\title{
CEVIAN SIMPLEXES
}

\section{SAHIB RAM MANDAN}

Inspired by the works of Court [3-10] in regard to cevian triangles and tetrahedra, it is proposed to introduce their analogues in higher spaces, establish a number of new results, and observe their interesting relations with the associated quadrics and $S$-configurations. The knowledge of the axioms of incidence in a projective space of $n$ dimensions, or briefly in an $n$-space, is assumed. The treatment is mainly synthetic and the analytic is suggested. The notations used here for intersections, joins, harmonic conjugacies, cross ratios, etc. follow Coxeter [11].

1. Introduction. a. Let $M$ be a point and $S=\left(A_{i}\right)$ a simplex in an $n$-space with vertices at the $n+1$ general points $A_{i}$ other than $M$; the $(n-1)$-dimensional simplex as well as the hyperplane of $S$ opposite $A_{i}$ be both denoted by its prime face $a^{i}$, its $(n-2)$-dimensional simplex and $(n-2)$-space opposite its edge $A_{i} A_{j}$ by its $(n-2)$-face $a^{i j}$, etc.; but for brevity, $A_{i} A_{j}$ be denoted by $a_{i j}$, the triangle $A_{i} A_{j} A_{k}$ as well as the plane $A_{k} a_{i j}$ of $S$ be both denoted by its plane face $a_{i j k}$, its tetrahedron $A_{i} A_{j} A_{k} A_{l}$ and the solid $A_{l} a_{i j k}$ by its solid face $a_{i j k l}$, etc.; $a^{i} \cdot A_{i} M=A_{i}^{\prime}, M a_{i j} \cdot a^{i j}=M^{i j}, M a^{i j} \cdot a_{i j}=M_{i j}, M a^{i j k} \cdot a_{i j k}=M_{i j k}$, $M^{i j k}=a^{i j k} \cdot M a_{i j k}, \cdots . S^{\prime}=\left(A_{i}^{\prime}\right)$ is then another simplex perspective to $S$ from $M$. If $p$ be an element of $S$ or associated with $S$, let the corresponding one of $S^{\prime}$ be denoted by $p^{\prime}$. Now by axioms of incidence only we have the following

Lemma 1. The $C_{2}^{n+1}$ joins $M_{i j} M^{i j}$, the $C_{3}^{n+1} M_{i j k} M^{i j k}, \cdots$, all concur at $M$ as the secants through $M$ to the corresponding pairs of opposite spaces of the simplex $S$. For every given value of $i$, the $n$ joins $A_{j} M^{i}$, the $C_{2}^{n} M_{j k} M^{i j k}, \cdots$, concur at $A_{i}^{\prime}$. For every given value of $i, j$, the $n-1$ joins $A_{k} M^{i j k}$, the $C_{2}^{n-1} M_{k l} M^{i j k l}$, etc. concur at $M^{i j} \ldots$. For every given value of $i, j, k, l$, the 4 joins $A_{i} M_{j k l}$ and the $3 M_{i j} M_{k l}$ concur at $M_{i j k l}$. For every given value of $i, j, k$, the 3 joins $A_{i} M_{j k}$ concur at $M_{i j k}$.

b. Definition 1. The joins $A_{i} A_{i}^{\prime}, M^{i j} M_{i j}, M^{i j k} M_{i j k}, \cdots$, are respectively said to be the cevians, bicevians, 3-cevians, etc. of $S . S^{\prime}$ is called the cevian simplex of $S$ for $M$, or of $M$ for $S$ which is then referred to as the anticevian simplex of $S^{\prime}$ for $M$ in analogy with such triangles and tetrahedra [4-7].

As an evident consequence we have another

Received by the editors October 26, 1959. 
Lemma 2. The cevians of all orders of a simplex $S$ through a point $M$ lie respectively along those of its cevian as well as anticevian simplex for $M$.

c. Lemma 3. The hyperplane $m$ of perspectivity of a simplex $S$ and its cevian simplex $S^{\prime}$ for a point $M$ coincides with the "polar" of $M$ for $S$ or $S^{\prime}$.

Proof. The 4 collinear triads of points $A_{i} M A_{i}^{\prime}, A_{j} M A_{j}^{\prime}, A_{i} A_{j}^{\prime} M^{i j}$, $A_{i}^{\prime} A_{j} M^{i j}$ are coplanar and form a quadrilateral $q$ whose diagonal triangle is $M_{i j}^{\prime} A_{i j} M_{i j}$ (\$1a), where $A_{i j}=a_{i j} \cdot a_{i j}^{\prime}=A_{i j}^{\prime}$. Now by the harmonic property of $q, H\left(A_{i} A_{j}, M_{i j} A_{i j}\right), H\left(A_{i}^{\prime} A_{j}^{\prime}, M_{i j}^{\prime} A_{i j}^{\prime}\right)$. Again the polar hyperplane $m[14 ; 15]$ of $M$ for $S$ or $S^{\prime}$ contains the $C_{2}^{n+1}$ points $A_{i j}=A_{i j}^{\prime}$. Hence the result.

2. Medial simplex. Let $G, G^{i}, G^{i j}, \cdots, G_{i j k l}, G_{i j k}$ be respectively the centroids of the simplex $S(\$ 1 \mathrm{a})$, its prime face $a^{i},(n-2)$-face $a^{i j}, \cdots$, tetrahedron $a_{i j k l}$, triangle $a_{i j k}$, and $G_{i j}$ be the midpoint of its edge $a_{i j} . A_{i} G^{i}, G^{i j} G_{i j}, G^{i j k} G_{i j k}, \cdots$, are then respectively its medians, bimedians, 3-medians, etc. and $\left(G^{i}\right)$ is its medial simplex evidently homothetic to it w.r.t. $G$ with $G G^{i}: G A_{i}=-1: n$ as their homothetic ratio [16]. Thus by Lemma 3 we have

LEMMA 4. The medial simplex of a given simplex is its cevian simplex for their common centroid, the hyperplane of their perspectivity being at infinity, and is therefore projectively equivalent to its cevian simplex for any other point.

3. Homology. Let $m$ be a hyperplane, $M$ a point outside it, and $k$ a constant. A point $P$ is said to transform into $P^{\prime}$ in the homology $\left(M, m,{ }^{\vee} k\right)$, if the biratio, cross ratio or anharmonic ratio $\left(P P^{\prime}, M Q\right)$ $=\left(P M \cdot P^{\prime} Q\right) /\left(Q P \cdot M P^{\prime}\right)=k$, where $Q=m \cdot M P$. If $m$ be at infinity, this homology becomes the homothety $(M, k)$ such that $P, P^{\prime}$ are homothetic w.r.t. $M$ with $k=M P / M P^{\prime}$ as its ratio. Now the cevian simplex $S^{\prime}$ of $S$ (\$1b) for $M$ becomes its medial, if $M$ lies at their common centroid $G(\$ 2)$ and consequently $m$ recedes to infinity. Thus we have

Theorem 1. A simplex in an $n$-space transforms into its cevian simplex $S^{\prime}$ for a point $M$ in the homology $(M, m,-n), m$ being the hyperplane of perspectivity of $S^{\prime}$ and the given simplex $S$.

\section{The converse proposition.}

Theorem 2. If the simplex $S$ and its elements be as in \$1a and $C_{2}^{n+1}$ points $M_{i j}$ be marked on its edges $a_{i j}$, one on each edge, such that the 3 
cevians $A_{j} M_{k i}$ of each of its $C_{2}^{n}$ plane faces $a_{i j k}$ through a vertex $A_{i}$ concur respectively at $M_{i j k}$, the same is true of its every triangle; the 4 cevians $A_{i} M_{j k l}$ and the 3 bicevians $M_{i j} M_{k l}$ of its every tetrahedron $a_{i j k l}$ concur respectively at $M_{i j k l} ; \cdots$; the $C_{2}^{n+1}$ hyperplanes $M_{i j} a^{i j}$ all CONCUR at $M$ where also CONCUR the $C_{3}^{n+1}(n-2)$-spaces $M_{i j k} a^{i j k}$, the $C_{4}^{n+1}(n-3)$-spaces $M_{i j k l} a^{i j k l}$, and so on.

Proof. Let $A_{i j}$ be the $C_{2}^{n+1}$ points on the edges $a_{i j}$ of $S$ such that $H\left(A_{i} A_{j}, M_{i j} A_{i j}\right)$. The 3 points $A_{i j}, A_{j k}, A_{k i}$ are then collinear in the harmonic or trilinear polar $m_{i j k}[7 ; 9 ; 11]$ of $M_{i j k}$ for the triangle $a_{i j k}$. The $C_{2}^{n}$ such lines lie in the hyperplane $m$ determined by the $n$ points $A_{i j}$ on its edges through $A_{i}$. Thus $m$ contains all the $C_{2}^{n+1}$ points $A_{i j}$. Hence $A_{i j}, M_{i j}$ form the $C_{2}^{n+1}$ pairs of opposite vertices of an $S$ configuration [14] such that the pole $M$ of $m$ for $S$ is one of the $2^{n}$ vertices of its dual thus satisfying the requisite conditions of the theorem by Lemmas 1 and 3 .

We may project $m$ to infinity to connect $M$ with $S$ as its centroid $G$ and be at ease for the rest of the proposition as the necessary consequence.

5. The cevian chain. a. The homological relation $(M, m,-n)$ of the 2 simplexes $S$ and $S^{\prime}(\S 3)$ discloses that we can derive either from the other, given $m$ or $M$. We may also repeat the construction in either direction to construct a cevian simplex $S^{\prime \prime}$ of $S^{\prime}$ as well as the anticevian ' $S$ of $S$ for $M$ or $m$.

Definition 2. $S^{\prime \prime}$ may be called the second cevian simplex of $S$ as well as the third cevian of ' $S$ for $M$ or $m$, and ' $S$ may be referred to as the second anticevian simplex of $S^{\prime}$ as well as the third anticevian of $S^{\prime \prime}$ for $M$ or $m$ (cf. [9]).

b. This construction of the chain of cevian as well as anticevian simplexes of $S$ for $M$ may be continued indefinitely and we may note the following properties in regard to them (cf. [9]):

(i) The polar hyperplane $m$ of $M$ for every simplex, or link, of the chain, is the same, and hence the same is true of its cevians of all orders (Lemma 2).

(ii) Any 2 links of the chain are perspective from $M$ with $m$ as the hyperplane of their perspectivity.

(iii) Of 2 consecutive links of the chain, one is inscribed in the other and forms its cevian simplex for $M$.

(iv) Any link of the chain may play the role of the initial one.

(v) Such a cevian chain is determined by a simplex and either a point $M$ or a hyperplane $m$.

(vi) Associated with a chain of cevian simplexes perspective from 
a point $M$ is a chain of $S$-configurations ( $\$ 4)$ such that they all have $C_{2}^{n+1}$ vertices common in their common hyperplane $m$ and their other vertices lie on the $C_{2}^{n+1}$ common bicevians of the simplexes, concurrent at $M$, one vertex of each configuration on each line.

c. Following Court [9] or making repeated use of the biratio of the homology of $\S 5 \mathrm{a}$ by projecting $m$ to infinity $(\S 3)$ we can prove

TheOREM 3. If $S, M, m$ be as in $\$ 3$, the ith cevian simplex of the the simplex $S$ for $M$ is its transform in the homology $\left(M, m,(-n)^{i}\right)$.

(This result provides a method to construct the cevian simplex of any order of $S$ for $M$ without constructing the intermediate ones.)

THEOREM 4. The biratio of 4 collinear vertices of 4 cevian simplexes of order $p, q, r, s$ of $S$ for $M$, or that of these 4 links of the chain of cevian simplexes perspective from $M$ and starting from $S$, is $\left(t^{p}-t^{r}\right)\left(t^{q}-t^{s}\right)$ $/\left(t^{p}-t^{s}\right)\left(t^{q}-t^{r}\right)$, where $t=-n$. Hence the biratio of any 4 consecutive links is the same constant, anywhere in the chain, equal to $(n-1)^{2}$ $/\left(n^{2}-n+1\right)$.

6. Cevian quadric. a. In an $n$-space $n(n+3) / 2$ general points always determine an $(n-1)$-quadric. Let $Q$ be one which touches the $n$ edges of the simplex $S(\$ 1 \mathrm{a})$ through a vertex $A_{i}$ at their respective points $M_{i j}$ and passes through the other $C_{2}^{n}$ points $M_{j k}$. The section of $Q$ by a plane face $a_{i j k}$ of $S$ is then a conic $q_{i j k}$ which touches its 2 edges $a_{i j}, a_{k i}$ respectively at $M_{i j}, M_{k i}$ and passes through $M_{j k}$. Now the 3 cevians $A_{i} M_{j k}$ of the triangle $a_{i j k}$ concur at $M_{i j k}$ by Lemma 1 . Then by Gergonne's theorem [8] $q_{i j k}$ touches its third side $a_{j k}$ at $M_{j k}$ with $M_{i j k}$ as its Gergonne point [10] for $q_{i j k}$. Thus the $C_{2}^{n}$ edges $a_{j k}$ of $S$, too, touch $Q$ at their respective points $M_{j k}$. Hence we have the following

THEOREM 5. There always exists a quadric $Q$ tangent to the edges of a simplex $S$ at the respective feet of its bicevians concurrent at a point $M$ [12].

Definition 3. $Q$ may be said to be the cevian quadric of $S$ for $M$ which may be referred to as the pole of contact of $Q$ for $S$. When $M$ lies at the centroid $G$ of $S, Q$ may be called the ellipsoid of Steiner, or briefly "eSt" of $S$, for then it cuts the plane faces of $S$ in their inscribed ellipses of Steiner $[8 ; 10]$.

b. Following Theorem 2 we may prove the converse proosition as

Theorem 6. If $C_{2}^{n+1}$ points be marked on the edges of a simplex $S$ in an n-space, one on each edge, such that there exist $C_{2}^{n}$ conics, one in each of its $C_{2}^{n}$ concurrent plane faces touching its 3 edges therein at their re- 
spectively marked points, the same is true of its every triangle and the $C_{3}^{n+1}$ such conics all lie on its cevian quadric $Q$ for a point $M$ such that the marked points are the feet of its bicevians through $M$.

As an evident consequence we have the following

Theorem 7. If $Q$ be the cevian quadric of the simplex $S$ for a point $M$, and $S$ along with its associated elements be as in $\$ 1 \mathrm{a}$, the section of $Q$ by a prime face $a^{i}$ of $S$ is its cevian $(n-2)$-quadric for $A_{i}^{\prime}, \ldots$, and that by a plane face $a_{i j k}$ of $S$ is its cevian conic $q_{i j k}$ for $M_{i j k \text {. }}$.

c. The $n$ points $M_{i j}$ on the $n$ edges of the simplex $S$ through a vertex $A_{i}$ determine the polar hyperplane $p^{i}$ of $A_{i}$ for the quadric $Q$ ( $\$ 6 \mathrm{a})$ by virtue of its construction. The $n$ points $M^{i j}(\S 1 \mathrm{a})$ determine the hyperplane $a^{i}$ of $S$. Now if $m$ ( $\left.\S 1 \mathrm{c}\right)$ be the hyperplane at infinity, $M$ lies at the centroid $G$ of $S, M^{i j}$ at the centroid $G^{i j}$ of its $(n-2)$ face $a^{i j}(\$ 2)$, and $M_{i j}$ at the midpoint $G_{i j}$ of its opposite edge $a_{i j}$ such that $G G_{i j}: G^{i j} G=(n-1): 2$, i.e., $p^{i}$ transforms into $a^{i}$ in the homothety $(G,(1-n) / 2)$. Thus by $\S 3$ we have the following

THEOREM 8. If $S, M, m$ be as in $\$ 3$ and $Q$ be the cevian quadric of the simplex $S$ for $M$, the polar simplex of $S$ for $Q$ transforms into $S$ in the homology $(M, m,(1-n) / 2)$. Consequently the polar hyperplane $m$ of $M$ for $S$ coincides with that for $Q$.

COROLlary 1. The $n+1$ vertices of $S$ together with $M$ form a selfconjugate $(n+2)$-ad of points for $Q$ such that the line joining any two of them is conjugate to the hyperplane containing the other $n$ points for $Q$ and consequently the polar $(n-p)$-space for $Q$ of the $(p-1)$-space containing any $p$ of them lies in the $(n-p+1)$-space containing the other $n-p+2$ points. Every one of these $n+2$ points is the center of perspectivity of the simplex formed of the other $n+1$ points and its polar for $Q[1$, Ex. 1, p. 218; 17].

COROLLARY 2. The center of the "eSt" of a simplex lies at its centroid (\$6c).

d. The collinearity of the points $M^{i j}, M, M_{i j}$ (\$1a) implies that their polar hyperplanes for the quadric $Q$ are coaxial. The polar $(n-3)$-space of $M^{i j}$ for the $(n-3)$-quadric section of $Q$ by the $(n-2)$ space $a^{i j}$ of the simplex $S$ lies in the polar hyperplane $m$ of $M$ for $Q$ as that for its $(n-2)$-face $a^{i j}$ by Theorems 7 and 8 . Thus we have

Theorem 9. If $S, M, M_{i j}, m, a^{i j}, a_{i j}$ be as in $\$ 1$ and $Q$ be the cevian quadric of the simplex $S$ for $M$, the tangent hyperplane of $Q$ at $M_{i j}$ is $\left(m \cdot a^{i j}\right) a_{i j}$. 
7. Ring contact of cevian and polar quadrics. a. The cevian conic $q_{i j k}$ of the triangle $a_{i j k}$ for $M_{i j k}(\$ 6 \mathrm{a})$ is the polar conic [11] of this point for its cevian triangle $M_{i j} M_{j k} M_{k i}$ [9]. (It may be remarked here that this is not true in a solid or higher spaces, i.e., the cevian quadric of a tetrahedron or a simplex for a point $M$ is not the polar quadric of $M$ for its cevian tetrahedron or simplex for $M$.) The polar line $m_{i j k}(\$ 4)$ of $M_{i j k}$ for either triangle coincides with that for $q_{i j k}$ and therefore with that for the polar conic $w_{i j k}$ of $M_{i j k}$ for $a_{i j k}$. Now Court [9] has shown that conics like $w_{i j k}, q_{i j k}$ belong to a pencil of doubly tangent conics, their common chord of contact being $m_{i j k}$. Again the $C_{3}^{n+1}$ conics like $q_{i j k}$ lie on the cevian quadric $Q$ of the simplex $S$ for $M(\S 6 \mathrm{a})$; the conics like $w_{i j k}$, each circumscribing the triangle $a_{i j k}$, are seen to lie on the polar quadric $W$, of $M$ for $S$, circumscribing $S$; the $C_{3}^{n+1}$ lines $m_{i j k}$ lie in the polar hyperplane $m$ of $M$ for $S, Q$, or $W$. Thus follows

Theorem 10. The cevian and the polar quadrics $Q, W$ of a point $M$ for a simplex $S$ have a "ring" contact along the polar hyperplane $m$ of $M$ for $S$ [2, p. 103].

b. Analytically it becomes almost obvious. For the equation of the cevian quadric $Q$ of the simplex $S$ for a point $M$ referred to $S$ may be at once put down as $\sum x_{i}^{2}-2 \sum x_{i} x_{j}=0$ [12], $M$ being the unit point $(1, \cdots, 1)$ of $S$. It is equivalent to $4 \sum x_{i} x_{j}-\left(\sum x_{i}\right)^{2}=0$ thus showing that $Q$ has ring contact with the polar quadric $W \equiv \sum x_{i} x_{j}=0$ of $M$ for $S$.

8. Associated $S$-configuration. We have seen in $\$ 4$ how the feet $M_{i j}$ of the bicevians of the simplex $S$ through $M$ on its edges and their "harmonics" $A_{i j}$ thereat form an $S$-configuration $(S-C)$ with $M$ as a vertex of its dual or reciprocal $(R \cdot S-C)$. The $(S-C)$ has $2^{n}$ hyperplanes, one of them being $m$, containing all the $A_{i j}$, as the polar of $M$ for $S$ [14]. Let $M_{8}$ be the other vertices of the $(R \cdot S-C)$ as the poles of the other hyperplanes $m^{s}$ of the $(S-C)$ for their common diagonal-simplex $S$. Every $m^{s}$ contains $C_{2}^{n+1}$ points $M_{i j}$ or $A_{i j}$ and is determined by $n$ such points on any $n$ concurrent edges of $S$, one on each edge. Hence the hyperplanes $p^{i}$ of the polar simplex of $S$ for its cevian quadric $Q(\S 6 \mathrm{c})$ coincide with $n+1$ hyperplanes of the $(S-C)$ (cf. [8]). We can now construct the cevian quadric $Q^{s}$ of $S$ for every $M_{s}$ and have

THEOREM 11. The cevian quadrics of a simplex $S$ in an $n$-space occur in sets of $2^{n}$ each such that the poles of contact of those of a set for $S$ form the vertices of the dual of an $S$-configuration $(S-C)$ with $S$ as 
their common diagonal simplex. Each quadric of the set touches the edges of $S$ at the vertices of the $(S-C)$ other than those lying in its corresponding hyperplane. The polar of each vertex of $S$ for every quadric of the set is a hyperplane of the $(S-C)$.

9. Pencil of cevian and polar quadrics. From Theorem 10 and the homological character of the links in a chain (\$5) of cevian simplexes perspective from a point $M$ follows the following

TheOREM 12. The cevian and the polar quadrics of a point $M$ for the links of a chain of cevian simplexes perspective from $M$ belong to $a$ pencil having a ring contact along the common hyperplane of perspectivity of the chain.

10. Bipunctual quadric. a. Hameed [12] proves analytically that the feet of 2 sets of bicevians, of a simplex $S$, concurrent respectively at 2 points $K, L$, on its edges lie on a quadric $U$. We can establish the same synthetically too by the method of $\$ 6 \mathrm{a}$ based upon the corresponding theorem for a triangle $[1$, Ex. 29, p. 53] as done by Court [6] for a tetrahedron and pointed out by Hameed himself [12]. The harmonically conjugate [2] or harmonic [12] quadric of $U$ splits up into the pair of polar hyperplanes of $K, L$ for $S$. Thus we have

Theorem 13. If a quadric $U$ passes through the feet of a set of concurrent bicevians of a simplex $S$ on its edges, $U$ meets them again in the feet of another set of concurrent bicevians of $S$. If $n$ pairs of points of $U$ on $n$ concurrent edges of $S$ be "isotomic" (i.e., equidistant from their respective midpoints), all such pairs behave alike, and $U$ then becomes the "eSt" of $S$ in the limit when these pairs of points coincide on their respective edges (\$6a).

CoROLlaRy. If $K_{i j}, L_{i j}$ be pairs of isotomic points on the edges of a simplex $S$ such that $K_{i j}$ are the feet of its bicevians concurrent at $K, L_{i j}$, too, lie at the feet of its bicevians concurrent at $L$ (say).

Definition 4. $K, L$ in this corollary may be referred as a pair of isotomic conjugate, or briefly isotomic, points for $S$ in analogy with such points for a triangle [7], and their polars for $S$ as a pair of isotomic hyperplanes for $S$. The quadric like $U$ in general may be said to be bipunctual for $S$ w.r.t. the pair of points $K, L$ in analogy with such a conic for a triangle $[1 ; 13]$.

b. We may thus develop the theory of the cevian quadric $Q$ of a simplex for a point $M$ as a limit of its bipunctual quadric $U$ w.r.t. a pair of points $K, L$ when $K, L$ coincide at $M$ [12]. 
11. Special simplexes. a. Following Court [3] and making use of Theorem 6 we can now prove the following

THEOREM 14. If $S=\left(A_{i}\right)$ be the simplex with vertices at the centers of $n+1$ hyperspheres, in an $n$-space, which touch one another externally, the $C_{2}^{n+1}$ points $M_{i j}$ of their contact lie on the hypersphere $(R)$ orthogonal to the given hyperspheres such that their $C_{2}^{n+1}$ centers of similitude $A_{i j}$ lie in a hyperplane $m$. The pole $M$ of $m$ for $S$ is the point for which $(R)$ is the cevian quadric of $S$. The $C_{2}^{n+1}$ midpoints of the segments $A_{i j} M_{i j}$ lie in the "Newton hyperplane" $p$ of the $S$-configuration with vertices at $A_{i j}, M_{i j}$. The radical hyperplane of $(R)$ and the circumhypersphere $(S)$ of $S$ coincides with $p[14 ; 19]$.

b. If a simplex $S$ be orthogonal or orthocentric with orthocenter at $H$, the $n-1$ altitudes of its $n-1$ plane faces through an edge $a_{i j}$ to $a_{i j}$ concur at the foot $M_{i j}$ on $a_{i j}$ of its bialtitude to $a_{i j}$ [18]. Hence by Theorems 6 and 13 we have the following

THEOREM 15. There exists a quadric $Q$ touching the edges of an orthogonal simplex $S$, in an $n$-space, at the feet thereat of its bialtitudes as its cevian quadric for its orthocenter $H$. The 9-point circles of its plane faces lie on its "first $n(n+1)$ point-sphere" $U$ as its bipunctual quadric w.r.t. $H$ and its centroid $G$. The radical hyperplane $h$ of $U$ and its circumhypersphere $(S)$ coincides with the polar of $H$ for $S$ or $Q$ as well as with that of $G$ for the polar hypersphere $(H)$ of $S$. $G H$ is therefore a normal to $h$, i.e., $h$ is normal to the "Euler line" of $S[18$ ].

Thanks are due to Professor B. R. Seth for his generous, kind and constant encouragement in my work.

\section{REFERENCES}

1. H. F. Baker, Principles of geometry, vol. 2, Cambridge, University Press, 1930.

2. - Principles of geometry, vol. 4, Cambridge, University Press, 1940.

3. N. A. Court, Sur quatre sphères tangents deux d deux, Mathesis vol. 42 (1933) pp. 227-228, 350-352.

4. - Modern pure solid geometry, New York, Macmillan \& Co., 1935. 89-91.

5. ——, On the cevian tetrahedron, Amer. Math. Monthly vol. 43 (1936) pp.

6. - Skewly cevian tetrahedrons, Duke Math. J. vol. 15 (1948) pp. 49-54.

7. - College geometry, New York, Barnes \& Noble, 1952.

8. - Sur les tétraèdres circonscrits par les arêtes à une quadrique, Mathesis vol. 63 (1954) pp. 12-18.

9. - The cevian chain, Scripta Math. vol. 22 (1956) pp. 193-202.

10. - Sur la transformation isotomique, Mathesis vol. 66 (1957) pp. 291-297.

11. H. S. M. Coxeter, The real projective plane, Cambridge, University Press, 1955.

12. A. Hameed, $A$ quadric associated with two points, Pakistan J. Sci. Res. vol. 3 (1951) pp. 49-52. 
13. S. R. Mandan, Feuerbach's theorem, Math. Student vol. 11 (1943) p. 25.

14. - An S-configuration in Euclidean and elliptic n-space, Canad. J. Math. vol. 10 (1958) pp. 489-501.

15. - Harmonic inversion, Math. Mag. vol. 32 (1959) pp. 71-78.

16. - Medial simplex, Math. Student (to appear).

17. - Polarity for a quadric in an n-space, J. Fac. Sci. Uni. Istanbul (to appear).

18. - Altitudes of a simplex in an n-space, J. Australian Math. Soc. (to appear).

19. - On n+1 intersecting hyperspheres in an n-space, J. Australian Math. Soc. (to appear).

INDIAN InSTITUTE OF TECHNOLOGY, KHARAgPUR

\section{GENERALIZATION OF COHN-VOSSEN'S THEOREM}

\section{CHIN-SHUI HSÜ}

In this paper Cohn-Vossen's Theorem $[1 ; 2$, pp. 127-133] is extended to a characterization of similarity in $E^{3}$. All surfaces here concerned are assumed to be orientable, closed, convex and of class $C^{3}$. All homeomorphisms between surfaces are assumed to be differentiable. A scalar $C^{2}$ function on a surface is harmonic if it satisfies the Laplace equation

$$
\Delta(\phi)=0
$$

where $\Delta$ is the second differential operator of Beltrami.

LEMma 1. (Hopf-Bochner $[3 ; 4]$ ). The only harmonic function defined on a surface is constant.

Lemma 2. Given two surfaces $S, \bar{S}$ and a homeomorphism $h: S \rightarrow \bar{S}$ where $h$ is conformal, the ratio of the first fundamental forms $\rho=\bar{I} / I$ satisfies

$$
\Delta(\log \rho)=2(K-\rho \bar{K})
$$

where $K, \bar{K}$ are the Gaussian curvatures.

Proof. Since the quantities on both sides of the equation are scalars it needs only to verify in a particular system of coordinates. For $C^{3}$ surfaces isothermal coordinates exists locally [5]. By employing such coordinates the verification is straightforward.

Received by the editors December 28, 1959. 